\title{
COMPARISON OF MIP MODELS FOR THE INTEGRATED LOT-SIZING AND ONE-DIMENSIONAL CUTTING STOCK PROBLEM
}

\author{
Gislaine Mara Melega ${ }^{1 *}$, Silvio Alexandre de Araujo ${ }^{1}$ and Raf Jans ${ }^{2}$ \\ Received November 30, 2015 / Accepted April 1, 2016
}

\begin{abstract}
Production processes comprising both the lot-sizing problem and the cutting stock problem are frequent in various industrial sectors. However these problems are usually treated separately, which can generates suboptimal overall solution and consequently causes production losses. In this paper, we propose different mathematical models for the integrated problem combining alternative models for the lot-sizing and the cutting stock problem, in order to evaluate and indicate the impact of these changes on the models' performance. An extensive computational study is done using randomly generated data and as a solution strategy we used a commercial optimization package and the application of a column generation technique.
\end{abstract}

Keywords: Capacitated Lot-Sizing Problems, One-Dimensional Cutting Stock Problem, Column Generation.

\section{INTRODUCTION}

Technological advances and increasing competition in industries have become worldwide phenomena. Some prominent aspects of these advances are the emphasis on knowledge and the development of new technologies. Inserted on this context, mathematical models that describe and improve the production processes have been studied and used as tools to support decision making in the business environment. Among the various decision processes, this work is part of the tactical/operational planning of production with the lot-sizing problem and cutting stock problem. The Lot-Sizing Problem ( $L S P$ ) considers the tradeoff between the setup and inventory costs to determine at minimal cost the size of production lots to meet the demand of each final product, while the cutting stock problem (CSP) involves the cutting of large objects into smaller items, so as to minimize the total loss of material.

The literature mostly deals with these two problems separately. However, in industrial sectors such as furniture, paper and aluminum, the lot-sizing and cutting stock problems are found in

\footnotetext{
*Corresponding author.

${ }^{1}$ Departamento de Matemática Aplicada, IBILCE, UNESP - Universidade Estadual Paulista, 15054-000 São José do Rio Preto, SP, Brasil. E-mails: gislainemelega@gmail.com; saraujo@ibilce.unesp.br

${ }^{2}$ HEC Montréal and CIRRELT, Canada H3T 2A7 QC, Canada. E-mail: raf.jans@ hec.ca
} 
consecutive phases. For these cases, a decoupled vision of the problems usually used by companies, can provide good local solutions but, at the end of planning processes, these solutions may conflict with the global objectives and feasibility of production. This fact was observed in Gramani et al. (2009) in the furniture industry. In this case, solutions generated from the lotsizing problem can lead to infeasible solutions for the cutting stock problem with regard to the production capacity of the cutting machine. If, on the other hand, for a given period, the production exceeds demand, this circumstance may generate cutting patterns with less loss, and a decrease of setup costs. However, there is an increase of inventory costs and physical space for allocation of items to be stored will be needed, which may not exist in practice. For this reason, the relevance of these issues in the industrial sectors and the benefits in dealing with problems in an integrated way makes this an appropriate research topic today. Motivated by this, the present paper proposes mathematical models and solution methods for solving the integrated lot-sizing and cutting stock problem.

In this work, the capacitated lot-sizing problem is modeled using the mathematical model proposed by Trigeiro et al. (1989), denoted here by $C L$. We also considered the variable redefinition strategy (Eppen \& Martin, 1987) which reformulates the lot-sizing problem as a shortest path problem. This reformulation is called SP. The idea of this variable redefinition was originally proposed for uncapacitated problems and Jans \& Degraeve (2004), Jans (2009), Fiorotto \& de Araujo (2014) and Melega et al. (2013) extended this to cases with capacity constraints, related parallel machines, unrelated parallel machines and various plants, respectively. More information on reformulation for lot-sizing problems can be found in Denizel et al. (2008), which shows several theoretical and computational results between some reformulations.

To model the one-dimensional cutting stock problem, we consider three mathematical models from the literature. The first one is the model developed by Kantorovich (1960), here denoted by $K T$. This model is also called the generalized assignment formulation for CSP (Degraeve \& Peeters, 2003) and determines the best way to cut objects to meet the demand, minimizing the number of objects used. For this model, an upper bound on the number of objects is considered. The second model dealt with, and perhaps the best known among the academic community, is the one proposed by Gilmore \& Gomory $(1961,1963)$ denoted here by $G G$. This model produces good lower bounds when compared to the $K T$ model, however it has an large number of variables, which indicates the use of column generation to deal with this difficulty.

The third model was proposed by Valério de Carvalho (1999, 2002), here denoted VC. The authors propose an alternative mathematical model for the one-dimensional cutting stock problem based on an arc flow problem. Thus, finding a valid cutting pattern for the cutting stock problem is equivalent to finding a path in a directed acyclic graph. The authors also consider additional constraints to guarantee that demand is satisfied. This model is efficient in the sense that it presents a linear relaxation as good as the Gilmore and Gomory model.

Furthermore, the cutting stock models described above were originally proposed for a single object type so they are extended to consider several types of objects in stock ( $M O$ from multi- 
objects). In a second part of this study, these models are integrated into the uncapacitated lotsizing problem proposed by Wagner \& Whitin (1958), here called WW.

Next, in Section 2, there is a literature review with the major studies that address the lot-sizing and the cutting stock problem in an integrated way. The Sections 3, 4 and 5 describe the proposed integrated models with capacity constraints, several object types and a reformulation for the lotsizing, respectively. In Section 6, the solution strategies are described. The computational results are presented in Section 7. And finally, the conclusions are presented in Section 8.

\section{LITERATURE REVIEW}

The importance of addressing the problems in an integrated way has become increasingly evident in the literature and is closely related to industrial applications. Possibly Farley (1988) had been the first paper that treats these problems in an integrated way. The author addressed the problem in an clothing industry, where the problem involves irregular two-dimensional cuts. Hendry et al. (1996) conducted a case study on a copper components factory. The objective was to investigate alternative methods of generating production plans for casting in order to minimize costs and meet demand. Nonås \& Thorstenson $(2000,2008)$ studied the problem coupled with the addition of setup cost in a Norwegian company that produces off-road trucks. The company needed a production plan that minimizes the total cost in the cutting process and in the production line. Therefore, the authors proposed a model and different solution methods. Gramani \& França (2006) formulated a mathematical model for the integrated problem in a furniture industry and proposed a solution method based on an analogy with the shortest path problem. Computational results compared the proposed method to the method used in the industry. Poltroniere et al. (2008) studied the integrated problem applied to the paper industry. The authors analyzed the production process and proposed a mathematical model for machines operating in parallel and presented two heuristics based on Lagrangian relaxation considering the problems in a decoupled way.

Ghidini \& Arenales (2009) treated the coupled problem applied to the furniture industry. To solve the problem, which considers the final composition of the products, the authors proposed two heuristics based on the primal simplex method with column generation. A procedure for obtaining an integer solution has been proposed. Gramani et al. (2009) proposed a mathematical model that considers final products and a capacity constraint on the saw machine in a furniture industry. Two solution methods were proposed. One is a decomposition heuristic and the other one is a Lagrangian based heuristic, in which the resulting problem is decomposed into two subproblems. A smoothing heuristic to recover feasibility is used. Gramani et al. (2011) extend their model from 2009 to consider cut items inventory and this model decomposed into two, one for the lot-sizing problem and one for the cutting stock problem in order to portray the solution method that industries practiced. The integrated problem was solved using the column generation technique. The computational results showed that the column generation technique can obtain gains of more than $12.7 \%$, compared with the decomposition model used in practice. Also in the context of a furniture factory Santos et al. (2011) presented a mixed integer programming 
model for the integrated problem in the context of a furniture factory. The authors considered the cutting patterns used by industry and n-groups cutting pattern to solve the problem with a set of real data provided by the factory. An extension to Gramani et al. (2011) model was proposed in Vanzela et al. (2013) in order to simulate the practice of a small furniture factory.

Alem \& Morabito (2012) used robust optimization tools to solve the integrated problem in the furniture industry, where the production costs and demand of products are uncertain parameters. Computational tests were performed using real and simulated data. Leão \& Arenales (2012) studied a simplification of the model studied in Poltroniere et al. (2008). Two approaches have been studied: (i) for a single type of object and (ii) for various types of objects. Three mathematical models for each approach have been proposed and they used the column generation as the solution method. To obtain a feasible solution, the columns obtained with the column generation method are used and the resulting integer problem with this limited set of columns is solved.

Silva et al. (2014) presented two integer programming models for the integrated problem. In these models, both the bringing forward of items for production and also the stocking of reusable leftovers for the cutting process in later periods were allowed. The authors proposed two heuristics and evaluated the models through a computational study, checking the quality of the solutions when compared to work described in the literature. In more recent papers, Poldi \& de Araujo (2016) studied the multi-period cutting stock problem and Molina et al. (2016) proposed an integrated lot sizing and packing problem that is related to the problem studied in this paper.

Most of the studies in the literature that address the integrated lot-sizing and cutting stock problem study several cases of the problem in practice. In these studies, alternative formulations for the LSP and CSP are not tested in order to choose the formulation that best fits the problem and the data set. So, extending the ideas of Longhi et al. (2015) this paper tries to cover the gap by proposing alternative mathematical formulations, considering different features of the lot-sizing and cutting stock problem such as capacity constraints and several object types. Furthermore, through the use of extensive computational tests, it intends to evaluate and point out the impact of these changes in the performance of the models.

\section{MATHEMATICAL MODELS WITH CAPACITY CONSTRAINT}

In this section the lot-sizing problem proposed by Trigeiro et al. (1989) (CL model) is integrated with various cutting stock formulations as described above. The integrated lot-sizing and cutting stock model is a two level production model, with production of final products at the final level, and the cutting of the pieces at the preceding level. The proposed model assumes that final products can be kept in inventory, but pieces cannot be kept in inventory. Furthermore, the Bill of Material indicates that one final product requires exactly one piece, and each type of final product requires a different piece. Therefore, there is a one-to-one relationship between final products and pieces in the model.

This might be a relevant model in various industrial applications. A first relevant setting is present, for instance, in the furniture industry. There is a demand for the final products (wardrobe, 
for example) which creates demand for subassemblies or components (a door, for example) (Gramani \& França, 2006). After the cutting process, the cut items pass through another productive process (e.g. drilling and painting) before being ready to be assembled into a final product. Usually these other productive processes are considered the bottlenecks of the complete production process (Ghidini et al., 2007; Santos et al., 2011; Alem \& Morabito, 2013), and their capacity is considered in the model, whereas the final assembly is not considered in the model since it is not a bottleneck process. Ghidini et al. (2007) and Alem \& Morabito (2013) considered the drill machine capacity in terms of items. Santos et al. (2011) considered aggregated capacity constraints for all the other machines (except the cutting machine). For the one-dimensional case, considered in this paper, the same type of capacity constraints appear. For instance, in tubular furniture industries, the bending machine is a bottleneck of the production process and its capacity must be considered in the mathematical model.

A second potential practical background appears in industries where the cut items are directly transformed in end items, i.e., there is no need to assemble several items to compose a final product, but there is a production process between the cut items and the end items. The capacity constraint in terms of end items means that the capacity constraints are related to the final production process to transform the cut items into the final items.

Consider the following parameters and decision variables:

\section{Parameters:}

$T$ : number of time periods (index $t$ );

$I$ : number of items (index $i$ );

$s c_{i t}$ : setup cost of item $i$ in period $t$;

$h c_{i t}$ : unit holding cost of item $i$ in period $t$;

$s t_{i t}$ : setup time of item $i$ in period $t$;

$v t_{i t}$ : production time of item $i$ in period $t$;

cap $_{t}$ : capacity (in unit of time) in period $t$;

$d_{i t}$ : demand of item $i$ in period $t$;

$s d_{i t r}$ : sum of demand of item $i$ from period $t$ until period $r$;

$L$ : object length;

$l_{i}$ : length of item $i$;

co: fixed cost of an object.

Decision Variables:

$X_{i t}$ : production quantity of item $i$ in period $t$;

$S_{i t}$ : inventory for item $i$ at the end of period $t ; Y_{i t}$ : binary variable indicating the production or not of item $i$ in period $t$.

The first cutting model used to formulate the integrated problem, is the $K T$ model, which gives the model $C L K T$ from $C L+K T$. For this, consider the following data and variables specific to this model: 
$Q$ : number of objects available in stock (index $q$ );

$y_{q t}$ : binary variable that indicates whether object $q$ is used in period $t$;

$h_{i q t}$ : variable that indicates the amount of item $i$ cut from object $q$ in period $t$.

\section{Model CLKT}

$$
\min \sum_{t=1}^{T} \sum_{i=1}^{I}\left(s c_{i t} Y_{i t}+h c_{i t} S_{i t}\right)+\sum_{t=1}^{T} \sum_{q=1}^{Q} \operatorname{coy}_{q t}
$$

Subject to :

$$
\begin{array}{lr}
X_{i t}+S_{i, t-1}=d_{i t}+S_{i t} & \forall i, \forall t \\
X_{i t} \leq s d_{i t} Y_{i t} & \forall i, \forall t \\
\sum_{i=1}^{I}\left(s t_{i t} Y_{i t}+v t_{i t} X_{i t}\right) \leq C a p_{t} & \forall t \\
\sum_{i=1}^{I} l_{i} h_{i q t} \leq L y_{q t} & \forall q, \forall t \\
Q & \\
\sum_{q=1}^{Q} h_{i q t}=X_{i t} & \forall i, \forall t \\
Y_{i t}, y_{q t} \in\{0,1\} & \forall i, \forall q, \forall t \\
h_{i q t} \in \mathbb{Z}_{+} & \forall i, \forall q, \forall t \\
X_{i t}, S_{i t} \in \mathbb{R}_{+} & \forall i, \forall t
\end{array}
$$

The objective function (1) minimizes the machine setup costs, inventory holding costs and the cost of cutting objects. Constraints (2) are the demand constraints: inventory carried over from the previous period and production in the current period are available to be used to satisfy current demand and build up inventory. Constraints (3) force the setup variable to one if any production takes place in that period. Next, there is a constraint on the available capacity in each period (4). Constraints (5) are from the cutting stock problem and ensure that if object $q$ is used, the combination of the lengths of items that will be cut from it should not exceed its length. Constraints (6) are responsible for the integration of the lot-sizing and cut stock problem decision, that is, we have to cut a sufficient amount of items to meet the planned production amount. Finally, the set of constraints (7), (8) and (9) define the non-negativity and integrality conditions. It is known that the model $K T$ for the cutting stock problem has a weak linear relaxation and presents many symmetric solution (Valério de Carvalho, 2002).

Observe that the variables $X_{i t}$ and constraints (6) could be eliminated by replacing $X_{i t}$ by $\sum_{q=1}^{Q} h_{i q t}$ in the rest of the model. However to compare this model with other proposed models, the variable $X_{i t}$ and the constraints (6) are kept explicitly in the model. This remark is also valid for the other models presented below in the Sections 3 and 4. 
The second integrated formulation presented below (CLVC) uses the $V C$ model for the cutting stock problem, which is modeled as an arc flow problem. Consider a path in a directed acyclic graph $G=(V, A)$, with $V=\{0,1, \ldots, L\}$ and the set of arcs of the graph defined as $A=$ $\left\{(j, l) ; 0 \leq j<l<L\right.$ and $l-j=l_{i}$ for all $\left.i \leq I\right\}$. The losses in the object generated from the cutting process are represented in the graph by additional arcs between the vertices $(j, j+1)$ to $j=0, \ldots, L-1$. As decision variables for the integrated model $C L V C$ we have:

$f_{t}$ : flow through the network in period $t$;

$z_{j l t}$ : number of cutting patterns which have an item of size $(l-j)$ allocated at a distance $j$ from the beginning of the object in period $t$.

\section{Model CLVC}

$$
\min \sum_{t=1}^{T} \sum_{i=1}^{I}\left(s c_{i t} Y_{i t}+h c_{i t} S_{i t}\right)+\sum_{t=1}^{T} c o f_{t}
$$

Subject to :

$$
\begin{array}{lr}
X_{i t}+S_{i, t-1}=d_{i t}+S_{i t} & \forall i, \forall t \\
X_{i t} \leq s d_{i t T} Y_{i t} & \forall i, \forall t \\
\sum_{i=1}^{I}\left(s t_{i t} Y_{i t}+v t_{i t} X_{i t}\right) \leq C a p_{t} & \forall t \\
-\sum_{(0, l) \in A} z_{0 l t}=-f_{t} & \forall t \\
\sum_{(g, l) \in A} z_{g l t}-\sum_{(l, h) \in A} z_{l h t}=0 & \\
\sum_{(l, L) \in A} z_{l L t}=f_{t} & \\
\sum_{\left(h, h+l_{i}\right) \in A} z_{h, h+l_{i}, t}=X_{i t} & \forall t \\
Y_{i t} \in\{0,1\} & \forall i, \quad \forall t \\
z_{l h t}, f_{t} \in \mathbb{Z}_{+} & \forall i, \quad \forall t \\
X_{i t}, S_{i t} \in \mathbb{R}_{+} & \forall(l, h) \in A, \forall t
\end{array}
$$

The objective function (10) minimizes the machine setup and inventory costs of the items, as well as the cost of the flow through the network. The flow set for this problem represents the number of used objects (cutting patterns), since one flow unit defines a path, which in turn defines a cutting pattern.

Constraints (11), (12) and (13) refer to the lot-sizing problem and are defined as in CLKT. The set of constraints (14), (15) and (16) correspond to flow conservation constraints and are characteris- 
tic of the Valério de Carvalho model. Constraints (17) integrate the two problems and constraints (18), (19) and (20) are non-negativity and integrality constraints.

This model also presents many symmetric solutions, or alternatives that correspond to the same cutting patterns. For this reason, Valério de Carvalho (1999) presented some reduction criteria to eliminate some arcs, reducing the number of symmetric solutions without eliminating any valid cutting patterns from set $A$. This study applies the criterion consisting of allocating the items in order of decreasing length in each cutting pattern, that is, an item of length $i_{1}$ can only be placed after another item length $i_{2}$ if $i_{1} \leq i_{2}$, or at the beginning of the object. Another criterion also used does not allow starting with a cutting pattern with loss. Thus, the first arc of loss will be inserted in the graph at a distance from the beginning of the object representing the shortest item length.

The third model $C L G G$ combines the lot-sizing model $C L$ with the cutting stock model $G G$ (Gilmore \& Gomory, 1961, 1963), which has the following parameters and decision variables:

$J$ : set of cutting patterns (index $j$ );

$a_{i j}$ : parameter indicating the quantity of item $i$ cut according to the cutting pattern $j$;

$x_{j t}$ : variable indicating the number of objects cut according to cutting pattern $j$ in period $t$.

\section{Model $C L G G$}

$$
\min \sum_{t=1}^{T} \sum_{i=1}^{I}\left(s c_{i t} Y_{i t}+h c_{i t} S_{i t}\right)+\sum_{t=1}^{T} \sum_{j \in J} c o x_{j t}
$$

Subject to :

$$
\begin{array}{lr}
X_{i t}+S_{i, t-1}=d_{i t}+S_{i t} & \forall i, \forall t \\
X_{i t} \leq s d_{i t T} Y_{i t} & \forall i, \forall t \\
\sum_{i=1}^{I}\left(s t_{i t} Y_{i t}+v t_{i t} X_{i t}\right) \leq C a p_{t} & \forall t \\
\sum_{j \in J} a_{i j} x_{j t}=X_{i t} & \forall i, \forall t \\
Y_{i t} \in\{0,1\} & \forall i, \forall t \\
x_{j t} \in \mathbb{Z}_{+} & \forall j, \forall t \\
X_{i t}, S_{i t} \in \mathbb{R}_{+} & \forall i, \forall t
\end{array}
$$

The objective function (21) minimizes the sum of setup costs, inventory costs and cost related to the number of objects used in the cutting process. The sets of constraints (22), (23) and (24) refer to the lot-sizing problem and are defined as in the model CLKT. Constraints (25) link the cutting variable with the production variable and finally the constraints (26), (27) and (28) are non-negativity and integrality constraints on the variables. The model $G G$ is an extended model and can be obtained by applying Dantzig - Wolfe decomposition on the $K T$ and $V C$ models 
(Valério de Carvalho, 2002). As a consequence this model has a large amount of variables and is generally solved by column generation.

\section{MATHEMATICAL MODELS WITH SEVERAL TYPES OF OBJECTS}

For the mathematical models presented in this section, we integrate the model proposed by Wagner \& Whitin (1958), here denoted by $W W$ with the cutting stock models extended to consider several types of objects. The $W W$ model describes the uncapacitated lot-sizing problem. In this case, consider the following parameters and additional decision variables:

Parameters:

$K$ : number of object types available in stock (index $k$ );

$e_{k t}$ : planned supply of object type $k$ at the beginning of period $t$;

$L_{k}$ : object length of type $k$;

$c w$ : cost per unit of raw material waste.

Decision Variable:

$s_{k t}$ : number of objects of type $k$ stocked at end of period $t$.

The model described below (WWKTMO) models the cutting stock problem based on model $K T$, considering several types of objects $M O$. In the original variables a new index is inserted, which refers to the type of object to be cut.

Parameters:

$s e_{k t}$ : sum of planned supply of objects of type $k$ from period 1 to period $t\left(s e_{k t}=\sum_{a=1}^{t} e_{k a}\right)$; $q \in\left\{1, \ldots, s e_{k t}\right\}$, index for the available objects.

Decision Variables:

$y_{q k t}$ : binary variable that indicates whether object $q$ of type $k$ is used in period $t$;

$h_{i q k t}$ : quantity of item $i$ cut from object $q$ of type $k$ in period $t$.

\section{Model WWKTMO}

$$
\min \sum_{t=1}^{T} \sum_{i=1}^{I}\left(s c_{i t} Y_{i t}+h c_{i t} S_{i t}\right)+c w\left(\sum_{t=1}^{T} \sum_{k=1}^{K} \sum_{q=1}^{s e_{k t}} L_{k} y_{q k t}-\sum_{t=1}^{T} \sum_{k=1}^{K} \sum_{q=1}^{s e_{k t}} \sum_{i=1}^{I} l_{i} h_{i q k t}\right)
$$

Subject to:

$$
\begin{array}{lr}
X_{i t}+S_{i, t-1}=d_{i t}+S_{i t} & \forall i, \forall t \\
X_{i t} \leq s d_{i t T} Y_{i t} & \forall i, \forall t \\
\sum_{i=1}^{I} l_{i} h_{i q k t} \leq L_{k} y_{q k t} & \forall q, \forall k, \forall t \\
\sum_{k=1}^{K} \sum_{q=1}^{s e_{k t}} h_{i q k t}=X_{i t} & \forall i, \forall t
\end{array}
$$




$$
\begin{array}{lr}
e_{k t}+s_{k, t-1}=\sum_{q=1}^{s e_{k t}} y_{q k t}+s_{k t} & \forall k, \forall t \\
Y_{i t}, y_{q k t} \in\{0,1\} & \forall q, \forall i, \forall k, \forall t \\
h_{i q k t} \in \mathbb{Z}_{+} & \forall q, \forall i, \forall k, \forall t \\
X_{i t}, S_{i t}, s_{k t} \in \mathbb{R}_{+} & \forall i, \forall t
\end{array}
$$

The objective function (29) minimizes machine setup costs, inventory holding costs and waste cost in the cutting process. Constraints (30) and (31) refer to the lot-sizing problem and are defined as in the CLKT model. The set of constraints (32) ensure that if an object $q$ of type $k$ is used, the combination of the items that will be cut from it should not exceed its length. Constraint (33) is responsible for the integration of the decision variables of the lot-sizing and cutting stock problems. It ensures that a sufficient amount of items are cut to meet the demand. The set of constraints (34) ensure that the number of objects cut in each period does not exceed the availability in stock.

The integrated model presented below $(W W V C M O)$, is based on the $V C$ formulation to model the cutting stock problem. Since several object types are available in stock, we need to define the maximum object length $L_{\max }=\max _{\forall k}\left\{L_{k}\right\}$, corresponding to the numbers of nodes in the network. The following decision variables are also needed:

$f_{k t}$ : flow through the network in the period $t$ for the object type $k$;

$z_{j l t}$ : number of cutting patterns which have a item of size $(l-j)$ allocated at a distance $j$ from the beginning of the object used in period $t$.

\section{Model WWVCMO}

$$
\min \sum_{t=1}^{T} \sum_{i=1}^{I}\left(s c_{i t} Y_{i t}+h c_{i t} S_{i t}\right)+c w\left(\sum_{t=1}^{T} \sum_{k=1}^{K} L_{k} f_{k t}-\sum_{t=1}^{T} \sum_{i=1}^{I} \sum_{\left(l, l+l_{i}\right) \in A} l_{i} z_{l, l+l_{i}, t}\right)
$$

Subject to:

$$
\begin{array}{lr}
X_{i t}+S_{i, t-1}=d_{i t}+S_{i t} & \forall i, \quad \forall t \\
X_{i t} \leq s d_{i t T} Y_{i t} & \forall i, \quad \forall t \\
-\sum_{(0, l) \in A} z_{0 l t}=-\sum_{k=1}^{K} f_{k t} & \forall t \\
\sum_{(g, l) \in A} z_{g l t}-\sum_{(l, h) \in A} z_{l h t}=0 & l=\left\{1, \ldots, L_{\max }-1\right\} \backslash \bigcup_{a=1}^{K}\left\{L_{a}\right\}, \forall t \\
\sum_{\left(g, L_{k}\right) \in A} z_{g L_{k} t}=f_{k t} & \forall k, \forall t \\
\sum_{\left(h, h+l_{i}\right) \in A} z_{h, h+l_{i}, t}=X_{i t} & \forall i, \forall t
\end{array}
$$




$$
\begin{array}{lr}
e_{k t}+s_{k, t-1}=f_{k t}+s_{k t} & \forall k, \forall t \\
Y_{i t} \in\{0,1\} & \forall i, \forall t \\
z_{g l t}, f_{k t} \in \mathbb{Z}_{+} & \forall(g, l) \in A, \forall k, \forall t \\
X_{i t}, S_{i t} \in \mathbb{R}_{+} & \forall i, \forall t
\end{array}
$$

The objective function (38) minimizes machine setup costs and storage costs of the items, and waste cost of raw material. Constraints (39) and (40) are defined as in the CLKT model. The set of constraints (41), (42) and (43) correspond to the flow conservation constraints. Constraints (44) integrate the cutting stock and lot-sizing problem. Constraints (45) ensure that the stock of the different types of available objects is respected and constraints (46), (47) and (48) are non-negativity and integrality constraints.

The following formulation ( $W W G G M O$ ) models the cutting stock problem proposed by Gilmore \& Gomory (1961) with an extension for several objects, which has the following parameters and decision variables. Observe that an index that refers to the type of object is also needed.

Parameters:

$J_{k}$ : set of cutting patterns that use object type $k$ (index $j$ );

$a_{i k j}$ : quantity of item $i$ cut from object type $k$ according to cutting pattern $j$.

Decision Variable:

$x_{k j t}$ : number of objects of type $k$ cut using cutting pattern $j$ in period $t$.

\section{Model WWGGMO}

$$
\min \sum_{t=1}^{T} \sum_{i=1}^{I}\left(s c_{i t} Y_{i t}+h c_{i t} S_{i t}\right)+c w\left(\sum_{t=1}^{T} \sum_{k=1}^{K} \sum_{j \in J_{k}} L_{k} x_{k j t}-\sum_{t=1}^{T} \sum_{k=1}^{K} \sum_{j \in J_{k}} \sum_{i=1}^{I} l_{i} a_{i k j} x_{k j t}\right)
$$

Subject to:

$$
\begin{array}{lr}
X_{i t}+S_{i, t-1}=d_{i t}+S_{i t} & \forall i, \forall t \\
X_{i t} \leq s d_{i t T} Y_{i t} & \forall i, \forall t \\
\sum_{k=1}^{K} \sum_{j \in J_{k}} a_{i k j} x_{k j t}=X_{i t} & \forall i, \forall t \\
e_{k t}+s_{k, t-1}=\sum_{j \in J_{k}} x_{k j t}+s_{k t} & \forall k, \forall t \\
Y_{i t} \in\{0,1\} & \forall i, \forall t \\
x_{k j t} \in \mathbb{Z}_{+} & \forall k, \forall j, \forall t \\
X_{i t}, S_{i t} \in \mathbb{R}_{+} & \forall i, \forall t
\end{array}
$$


The objective function (49) minimizes machine setup cost and inventory cost of items, and costs of waste due to the cutting of objects. Constraints (50) and (51) refer to the lot-sizing problem. The set of constraints (52) is responsible for the integration of the decision variables of the two problems. The constraints (53) ensure that the number of cut objects in each period does not exceed the availability in stock, and the constraints (54), (55) and (56) are non-negativity and integrality constraints.

\section{INTEGRATED MODELS USING THE REFORMULATED LOT-SIZING PROBLEM SP}

The integrated models presented in Sections 3 and 4 use the classical formulation for the lotsizing problem. In order to obtain better lower bounds, the lot-sizing problem is reformulated as a shortest path problem (Eppen \& Martin, 1987) and then integrated into the respective cutting stock problem. For that, we need the following definitions of parameters and variables, respectively:

$c v_{i t r}$ : inventory holding cost of item $i$ in period $t$ at a quantity that meets the demands for the periods from $t$ until $r$;

$z v_{i t r}$ : fraction of the production plan for item $i$ to satisfy demand from period $t$ to period $r$.

The lot-sizing problem variables have the following correspondence:

$$
X_{i t}=\sum_{r=t}^{T} s d_{i t r} z v_{i t r} \quad \forall i, \forall t
$$

and the demand constraints (2) and setup constraints (3) are rewritten in terms of the new decision variables as follows:

$$
\begin{array}{lr}
\sum_{r=1}^{T} z v_{i 1 r}=1 & \forall i \\
\sum_{r=1}^{t-1} z v_{i r t-1}=\sum_{r=t}^{T} z v_{i t r} & \forall i, \forall t \backslash\{1\} \\
\sum_{r=t}^{T} z v_{i t r} \leq Y_{i t} & \forall i, \forall t
\end{array}
$$

Constraints (58) and (59) define the flow constraints in the SP model. For each item $i$, a unit flow is sent through the network (constraint (58)), imposing that its demand has to be satisfied without backlogging in each period (59)). Constraint (60)) ensures that item $i$ will be produced in period $t$ only if there is a setup prepared to produce that item.

Note that the constraint (58) forces production in the first period, which it is not a problem for the data set used in this work, because all the demand equal 0 in the used data set is replaced to be strictly greater than zero (equal 1 unit). Otherwise, if the demand for some item is zero for the first period, it would be necessary to slightly adapt the formulation (Pochet \& Wolsey, 2006; Jans \& Degraeve, 2004). 
Thus, we propose new mathematical models that integrate the cutting stock problems $(K T, V C$, $G G, K T M O, V C M O, G G M O$ ) into the reformulated lot-sizing problem $(S P)$. This is done by replacing the decision variables in the models, by the new variables and constraints described above.

As an example, the integrated model SPKT is shown next.

\section{Model SPKT}

$$
\min \sum_{t=1}^{T} \sum_{i=1}^{I}\left(s c_{i t} Y_{i t}+\sum_{r=t}^{T} c v_{i t r} z v_{i t r}\right)+\sum_{t=1}^{T} \sum_{q=1}^{Q} \operatorname{coy}_{q t}
$$

Sujeito a :

$$
\begin{array}{lr}
\sum_{r=1}^{T} z v_{i 1 r}=1 & \forall i \\
\sum_{r=1}^{t-1} z v_{i r t-1}=\sum_{r=t}^{T} z v_{i t r} & \forall i, \forall t \backslash\{1\} \\
\sum_{r=t}^{T} z v_{i t r} \leq Y_{i t} & \forall i, \forall t \\
\sum_{i=1}^{I}\left(s t_{i t} Y_{i t}+\sum_{r=t}^{T} v t_{i t} s d_{i t r} z v_{i t r}\right) \leq C a p_{t} & \\
\sum_{i=1}^{I} l_{i} h_{i q t} \leq L y_{q t} & \forall t \\
\sum_{q=1}^{Q} h_{i q t}=\sum_{r=t}^{T} s d_{i t r} z v_{i t r} & \forall q, \forall t \\
Y_{i t}, y_{q t} \in\{0,1\} & \forall i, \forall t \\
h_{i q t} \in \mathbb{Z}_{+} & \forall i, \quad \forall q, \forall t \\
z v_{i t r}, S_{i t} \in \mathbb{R}_{+} & \forall i, \forall t
\end{array}
$$

The objective function (61) minimizes the setup costs and inventory holding costs according to the $S P$ model, as well as, the cost of the number of objects to be cut. The constraints (62) and (63) define the flow constraints of the $S P$ model. The set of constraints (64) also refers to the model $S P$ and ensures that there will be production for an item $i$ in period $t$, only if the machine is setup to produce this item. Capacity constraints (65) ensure that the capacity available is not violated. Constraints (66) refers to the knapsack constraints in the $K T$ model. The set of constraints (67) integrates the two problems and finally (68), (69) and (70) are non-negativity and integrality constraints. 


\section{SOLUTION STRATEGIES}

Two heuristic strategies are proposed for solving the models described in sections 3, 4 and 5 . The first heuristic consists of solving the models integrated with $K T$ and $V C$ using a commercial optimization package with a stopping criteria. The solver is stopped at the end of 600 seconds or when the gap between the upper bound and lower bound is less than $0.1 \%$. The second heuristic proposes the search for a feasible solution by using the column generation technique for the models integrated with $V C$ and $G G$. The solver is used to solve the restricted master problems, the sub-problems and also to solve the resulting integer restricted master problem.

Next, the column generation based heuristic is explained in more detail together with the procedure to obtain a feasible solution.

\subsection{Initial Basic Solution}

Considering the model with capacity constraint and integrated with the $G G$ model, the master problem starts with the columns related to the homogeneous cutting pattern, i.e., columns of type: $\left(0, \ldots, a_{i i}, \ldots, 0\right)$, where $a_{i i}=\left\lfloor\frac{L}{l_{i}}\right\rfloor, \forall i$. The same columns are inserted for each period, $t=1, \ldots, T$.

For the problems with several objects, the master problem starts with homogeneous cutting patterns generated for each object type, i e., for each object $k$ columns of type $\left(0, \ldots, a_{i k i}, \ldots, 0\right)$, where $a_{i k i}=\left\lfloor\frac{L_{k}}{l_{i}}\right\rfloor, \forall i$, are inserted in order to avoid the infeasibility of an initial basic solution. The same columns are inserted for all periods. Similar ideas are applied to the models integrated with $V C$.

\subsection{Column Generation Procedure}

The sub-problems in the column generation consist of finding a new cutting pattern for the master problem. For the models integrated with $G G$, the sub-problem is composed of the knapsack constraint and for those integrated with $V C$ the sub-problem is composed of the flow constraints imposing a flow equal to one, which represents a cutting pattern.

Considering the models with one type of object and a capacity constraint, a sub-problem is solved for every period, and the column with the smallest reduced cost over all periods is included in the master problem. As a consequence, the same column is also inserted for each period. For the problems with several objects, a sub-problem is solved for each period and object type, and the column with the smallest reduced cost is inserted into the master problem. The same column is used for all periods according to the corresponding object in the restricted master problem.

\subsection{Feasibility Strategy}

The column generation part is stopped when the minimum reduced cost is non-negative for each subproblem or when the time limit is reached. The integer restricted master problem including 
all the columns obtained in the column generation procedure is solved using the optimization package with a time limit of 600 seconds and an optimality gap of $0.1 \%$.

Table 1 presents a summary of all proposed mathematical models and solution strategies.

Table 1 - Mathematical Models and Solution Strategies.

\begin{tabular}{|c|c|c|}
\hline Models & Considered Problems & Solution Strategies \\
\hline$C L K T$ & & \\
$S P K T$ & $L S P$ with capacity constraint & Optimization package \\
b $L V C$ & $C S P$ single object type & \\
$S P V C$ & & \\
\hline$C L G G_{-} C G$ & & \\
$S P G G_{-} C G$ & $L S P$ with capacity constraint & Column generation \\
bLVC_CG & $C S P$ single object type & based heuristic \\
$S P V C_{-} C G$ & & \\
\hline$W W K T M O$ & & \\
$S P K T M O$ & $L S P$ without capacity constraint & Optimization package \\
$W W V C M O$ & $C S P$ with several object types & based heuristic \\
$S P V C M O$ & & \\
\hline$W W G G M O \_C G$ & & Column Generation \\
$S P G G M O \_C G$ & $L S P$ without capacity constraint & based heuristic \\
$W W V C M O \_C G$ & $C S P$ with several object types & \\
$S P V C M O \_C G$ & & \\
\hline
\end{tabular}

\section{COMPUTATIONAL STUDY}

This section presents the computational results used to evaluate and compare the performance of the proposed models. The analysis of the results is done through tables containing the average values found by models with respect to different aspects, as well as the use of the performance profile technique (Dolan \& Moré, 2002). This technique provides a tool which facilitates the exhibition and the interpretation of comparisons and it is briefly described in the following paragraphs.

Consider $\mathbb{P}$ as the set of $\mathfrak{n}_{p}$ instances and $\mathbb{M}$ as the set of $\mathfrak{n}_{M}$ models described in sections 3, 4 and 5. The values obtained for each instance (upper bound and gap) $p \in \mathbb{P}$ using the $m \in \mathbb{M}$ model is denoted by $v_{p, m}$. For each model $m \in \mathbb{M}$ a comparison of its performance on the instance $p \in \mathbb{P}$ relative to the performance of the best model is given by the following performance ratio:

$$
r_{p, m}=\frac{v_{p, m}}{\min _{m \in \mathbb{M}}\left\{v_{p, m}\right\}} .
$$

If the model $m$ does not find a feasible solution for an instance $p$ then $r_{p, m}$ is defined as $r_{M}$, which is set at one unit more than the worst value of the performance ratio found for all the 
feasible instances in all the models. The performance of the model $m$ compared to the other models is given by the performance profile:

$$
\rho_{m}(\tau)=\frac{1}{\mathfrak{n}_{p}}\left|\left\{p \in \mathbb{P}: r_{p, m} \leq \tau\right\}\right|
$$

with $|$.$| representing the number of elements in the set. The performance profile \rho_{m}(\tau)$ is a function that is associated to a given value $\tau \in \mathbb{R}$, and indicates the fraction of instances solved by the model $m$ with a performance within a factor $\tau$ of the best performance found. With this, each model has a curve that shows its performance for each level $\tau$. Due to the fact that $r_{M}$ can be considerably large, the logarithm scale is used to represent the performance profile. It is done as follows (Dolan and Moré, 2002):

$$
\tau \mapsto \frac{1}{\mathfrak{n}_{p}}\left|\left\{p \in \mathbb{P}: \log _{2}\left(r_{p, m}\right) \leq \tau\right\}\right| .
$$

This way, the $\tau$ factor varies in $\left[0, r_{M}\right)$, with $r_{M}=1+\max \left\{\log _{2}\left(r_{\bar{p}}, \bar{m}\right): \bar{p} \in \mathbb{P}\right.$ and $\left.\bar{m} \in \mathbb{M}\right\}$.

The models are written in the AMPL syntax (Fourer et al., 1990) and CPLEX 12.5 (IBM, 2009) is used as solver. All the computational tests are conducted on a $2.93 \mathrm{GHz}$ Intel Core $i 7$ processor with $8 \mathrm{~GB}$ of RAM memory.

\subsection{Experiment 1}

Next, we describe the data generation and computational results for the integrated problem with capacity constraints (models in Section 3 and the corresponding models in Section 5). In this experiment we present two sets of data: the first one called Data 1, is based on a data generator for the cutting stock problem. The second set, referred to as Data 2, is based on some examples widely used in the literature to solve the lot-sizing problem.

\subsubsection{Data Sets}

For the Data 1 set, the CUTGEN1 generator proposed by Gau \& Wäscher (1995) is used and for the lot-sizing problem the data set is based on Trigeiro et al. (1989). The parameters are generated in intervals $[a, b]$ with a uniform distribution as follows:

- number of periods: $T=15$

- object length: $L=1000$

- number of items: $I=\{10,20,40\}$

- length of items are generated in three intervals according to $l_{i} \in\left[v_{1}, v_{2}\right]$, with $v_{1}=$ $0.01 L$ or $0.2 L$ and $v_{2}=0.02 L$ or $0.8 L$

- demand for items is generated according to the idea of Gau \& Wäscher (1995) for a single period, so that the average demand over all items in a specific period is equals 100 . 
- raw material cost: $c o=1$;

- setup cost: $s c_{i t} \in[100,500]$

- inventory cost: $h c_{i t} \in[1,5]$

- production time: $v t_{i t}=1$

- setup time: $s t_{i t} \in[10,50]$

- capacity $\left(\mathrm{cap}_{t}\right)$ is generated by the average of lot-by-lot policies: for every period $t$ calculate the amount of resources needed to produce exactly the demands of the items in this period, sum up this amount for all periods and divide by the number of periods $T$, this is, $\operatorname{cap}_{t}=\frac{\sum_{t=1}^{T} \sum_{i=1}^{I}\left(v t_{i t} d_{i t}+s t_{i t}\right)}{T}$.

In the cutting stock problem modeled by $K T$, an upper bound on the number of objects in stock $(Q)$ is needed. Poldi \& Arenales (2010) is the basis for calculating the upper bound as $Q=2\lceil\lambda\rceil$ with, $\lambda=\frac{\sum_{t=1}^{T} \sum_{i=1}^{I} l_{i} d_{i t}}{L}$.

Thus, the Data 1 defines 9 classes (see Table 2), and for each class 10 random instances are generated considering the three levels of capacity, totaling 270 instances.

Table 2 - Classes of Data 1.

\begin{tabular}{|c|cc|}
\hline \multirow{2}{*}{ Classes } & \multicolumn{2}{|c|}{ Data 1} \\
\cline { 2 - 3 } & Items & $v_{1} ; v_{2}$ \\
\hline Class 1 & 10 & $0.01 ; 0.2$ \\
Class 2 & 10 & $0.01 ; 0.8$ \\
Class 3 & 10 & $0.2 ; 0.8$ \\
\hline Class 4 & 20 & $0.01 ; 0.2$ \\
Class 5 & 20 & $0.01 ; 0.8$ \\
Class 6 & 20 & $0.2 ; 0.8$ \\
\hline Class 7 & 40 & $0.01 ; 0.2$ \\
Class 8 & 40 & $0.01 ; 0.8$ \\
Class 9 & 40 & $0.2 ; 0.8$ \\
\hline
\end{tabular}

The second data set, called Data 2, is based on the lot-sizing problem. This set is created using 7 specific instances from Trigeiro et al. (1989) (see Table 3). These instances are considered difficult and have been used in several studies in the literature (Jans \& Degraeve, 2004; Vyve \& Wolsey, 2006; Degraeve \& Jans, 2007; de Araujo et al., 2015).

To complete the data file the CUTGEN1 is used as generator for the cutting stock parameters and it was described above. Thus, Data 2 defines 3 classes (see Table 3), and for each class 10 random instances are generated for each of the 7 specific instances, totaling 210 instances. 
Table 3 - Specific Instances and Classes of Data 2.

\begin{tabular}{|c|cc|}
\hline Instance & Items & Periods \\
\hline G30 & 6 & 15 \\
G30wol & 6 & 15 \\
G53 & 12 & 15 \\
G57 & 24 & 30 \\
G62 & 6 & 30 \\
G69 & 12 & 30 \\
G72 & 24 & 30 \\
\hline
\end{tabular}

\begin{tabular}{|c|c|}
\hline \multirow{2}{*}{ Classes } & Data 2 \\
\cline { 2 - 2 } & $v_{1} ; v_{2}$ \\
\hline Class 10 & $0.01 ; 0.2$ \\
Class 11 & $0.01 ; 0.8$ \\
Class 12 & $0.2 ; 0.8$ \\
\hline
\end{tabular}

\subsubsection{Computational Results}

Firstly, we present the computational results using Data 1.

A preliminary test is performed in order to evaluate the impact of the models and the capacity tightness on the number of feasible solutions found. It is considered three different levels of capacity: cap 0 where we have the integrated problem without capacity constraint; cap 1 where the value of the capacity is $c a p_{t} / 0.3$; cap 2 where we have tighter capacity given by $c a p_{t} / 0.85$. The results shown that the number of feasible solutions decreases as the capacity becomes more scarce. The CLVC model is the only formulation able to find feasible solutions for all instances, but only for the uncapacitated case. The $C L V C$ model obtained the largest number of feasible solutions for the three capacity levels using the optimization package based heuristic. For the column generation based heuristic the best performance in this analysis is obtained by CLVC_CG model. In general, the difficulty to find a feasible solution is in Classes 4 and 7, which correspond to classes in which the ratio between the item length and object length is small.

The following results are evaluated considering the uncapacitated model (column cap 0 ) and with cap $_{t} / 0.3$ (column cap 1 ).

In Table 4 the lower bound obtained with the linear relaxation for the models is presented. As a general conclusion, regarding the cutting stock problem, the models integrated with $V C, V C \_C G$ and $G G_{-} C G$ are equivalent with relation to the value of the lower bound obtained. For this reason just one of them is presented and the lower bounds are better than the lower bounds resulting from the models integrated with $K T$. The value obtained with cap 0 and cap 1 are the same for all the models and just one value is presented. Regarding the lot sizing problem the models integrated with SP obtain a substantially better lower bound than the models using $C L$.

Figures 1 and 2 show the performance profiles obtained with the upper bound for all the instances in Data 1. Considering the optimization package based heuristic (see Fig. 1), the CLVC model shows a good overall performance in both variations of capacity, since its performance profile dominated the rest of them. This model could find the best upper bound for around $70 \%$ of the instances, followed by the SPVC. For the column generation based heuristic (see Fig. 2), CLVC_CG obtained the best performance for around $50 \%$ of the instances, followed by SPGG_CG. Just 
Table 4 - LP Relaxation for Data 1.

\begin{tabular}{|c|c|c|c|c|}
\hline Classes & CLKT & SPKT & CLVC & SPVC \\
\hline Class 1 & 10978.63 & 34793.33 & 10978.65 & 34794.62 \\
Class 2 & 15735.78 & 39828.10 & 16801.00 & 41005.42 \\
Class 3 & 17110.47 & 41202.79 & 18733.81 & 42964.73 \\
Class 4 & 22083.04 & 70486.11 & 22083.04 & 70486.11 \\
Class 5 & 31493.67 & 79562.22 & 33074.99 & 81292.92 \\
Class 6 & 34020.87 & 82345.81 & 36519.61 & 85032.61 \\
Class 7 & 43091.46 & 137835.25 & 43091.46 & 137835.25 \\
Class 8 & 59926.51 & 154188.53 & 61315.45 & 155838.87 \\
Class 9 & 65761.36 & 159574.61 & 68065.34 & 162285.77 \\
\hline Average & 33355.75 & 88868.53 & 34518.15 & 90170.70 \\
\hline
\end{tabular}

considering the cap0 instances it is possible to obtain an upper bound for all the instances with the $C L V C, C L V C_{-} C G$ and $C L G G_{-} C G$ models. The lowest number of feasible solutions, around $10 \%$ and $40 \%$, are found respectively by the SPKT and SPVC_CG models. In general, mathematical models integrated into $V C$ obtained the best results for most instances in both the capacity variations and heuristic strategies.

Figure 3 shows the performance profile obtained for the gap for models solved with the optimization package based heuristic. The gap is that provided by the solver for the final solution for both the cases cap 0 and cap1. In both variations of capacity, the SPKT could not find a satisfactory results for the gap. The CLVC model showed a good overall performance, in which $100 \%$ and $90 \%$ of the instances obtained a feasible solution for cap 0 and cap 1 , although it has the best performance in just $30 \%$ of the instances. On the other hand, the SPVC model showed the best performance in approximately $60 \%$ of the instances, but with the disadvantage of not having solved all the instances. Since the column generation procedure is subject to a time limit, we cannot guarantee the optimality of this lower bound, and therefore no results on the gap are presented for the $C G$ methods.

The computational results for Data 2 are given as follow.

The same analysis for Data 2 is performed in order to evaluate the impact of the models and the capacity tightness on the number of feasible solutions found. It is noticed a big difficulty of the SPKT model to solve these instances. The SPVC_CG model also shows great difficulties in finding a feasible solution. Class 12 presents the largest number of feasible solutions (378 from 560), followed by Class 11 (369 from 560). In Class 12, half of the models found a feasible solution for all the instances; this class is related with the longest items when compared to object length.

In the Table 5 the lower bound obtained with the linear relaxation for the models solved with the optimization package are presented. As discussed above the models $V C, V C_{-} C G$ and $G G_{-} C G$ are equivalent with relation to the lower bound obtained and due to this reason just one of them is presented. The use of a reformulated lot-zing model once again, improves the results compared 

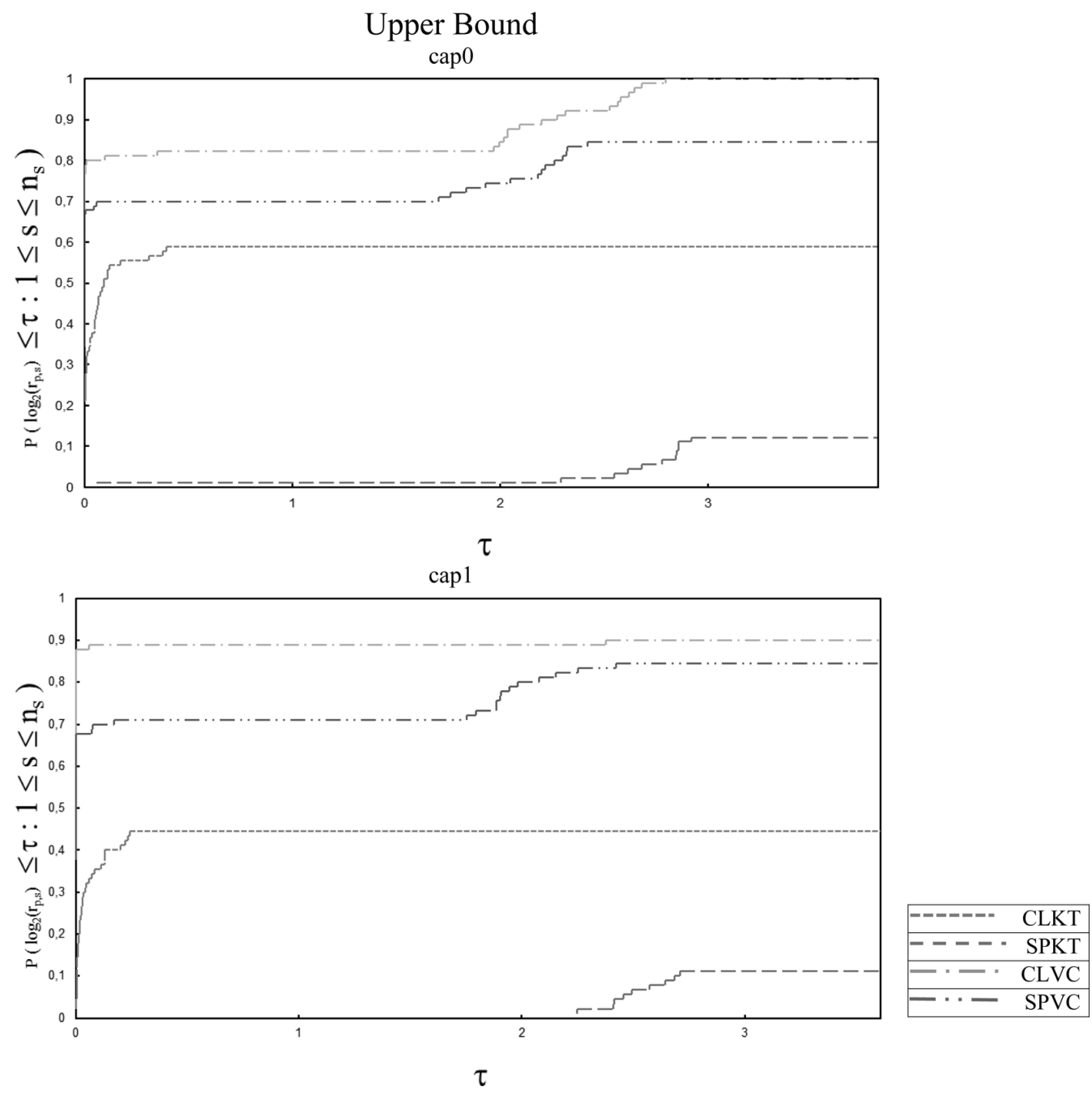

Figure 1 - Performance Profiles: Upper Bound for the Optimization Package Based Heuristic - Data 1.

to the models integrated with the classical lot-zing model. This improvement is $260 \%$ on average. The models integrated with $K T$ did not achieve a value for the linear relaxation for some instances within a time of 5 hours.

Figure 4 shows the performance profile of the upper bound and gap considering the optimization package based heuristic. The first impact observed in the figure is due to the huge difference between the models integrated with $K T$ and $V C$ to obtain an upper bound. The SPKT and CLKT models found an upper bound for around just 5\% and $40 \%$ of the instances, respectively. On the other hand, the SPVC and CLVC models found a upper bound for around $65 \%$ and $90 \%$ of the instances, respectively. The best performance to obtain the upper bounds is found using the CLVC model with more than $80 \%$ of the instances $(\tau=0)$, compared with the other models, 

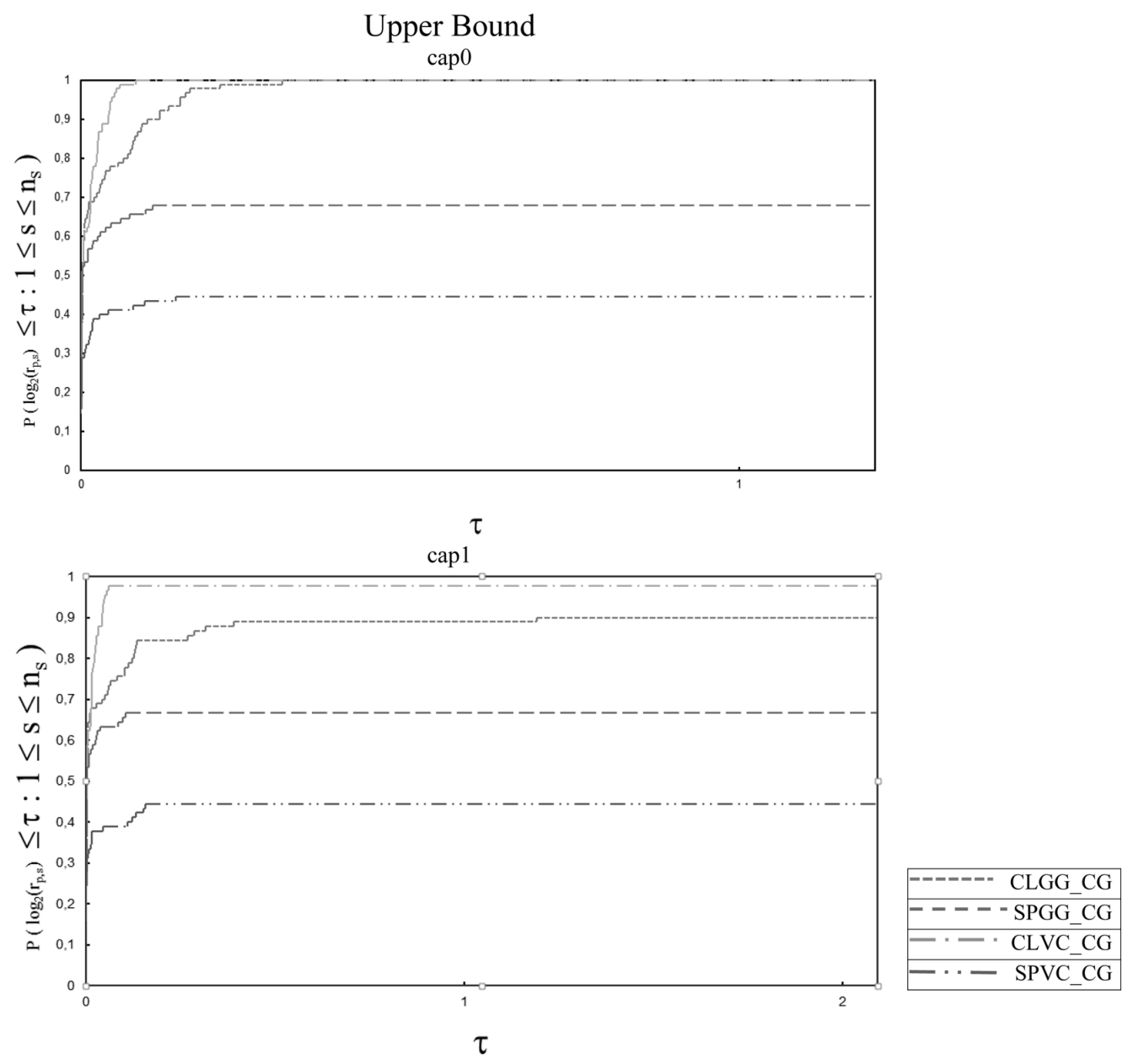

Figure 2 - Performance Profiles: Upper Bound for the Column Generation Based Heuristic - Data 1.

which reached at most $10 \%$ of the best results (at $\tau=0$ ). The performance obtained with the $S P V C$ models changes a little when the gap is analyzed. The performance of the SPVC becomes around the same as $C L V C, 40 \%$ of the best results $(\tau=0)$. And one more time, results obtained with $C L V C$ model dominate the other models considering the performance values $(\tau)$.

Figure 5 presents the performance obtained in the column generation based heuristic for the upper bounds. We can see that the CLVC_CG model found an upper bound for around $98 \%$ of the instances, although it could reach the best performance for just $20 \%$ of the instances. On the other hand, CLGG_CG found the best results for the upper bound for almost $75 \%$ of the instances solved. 

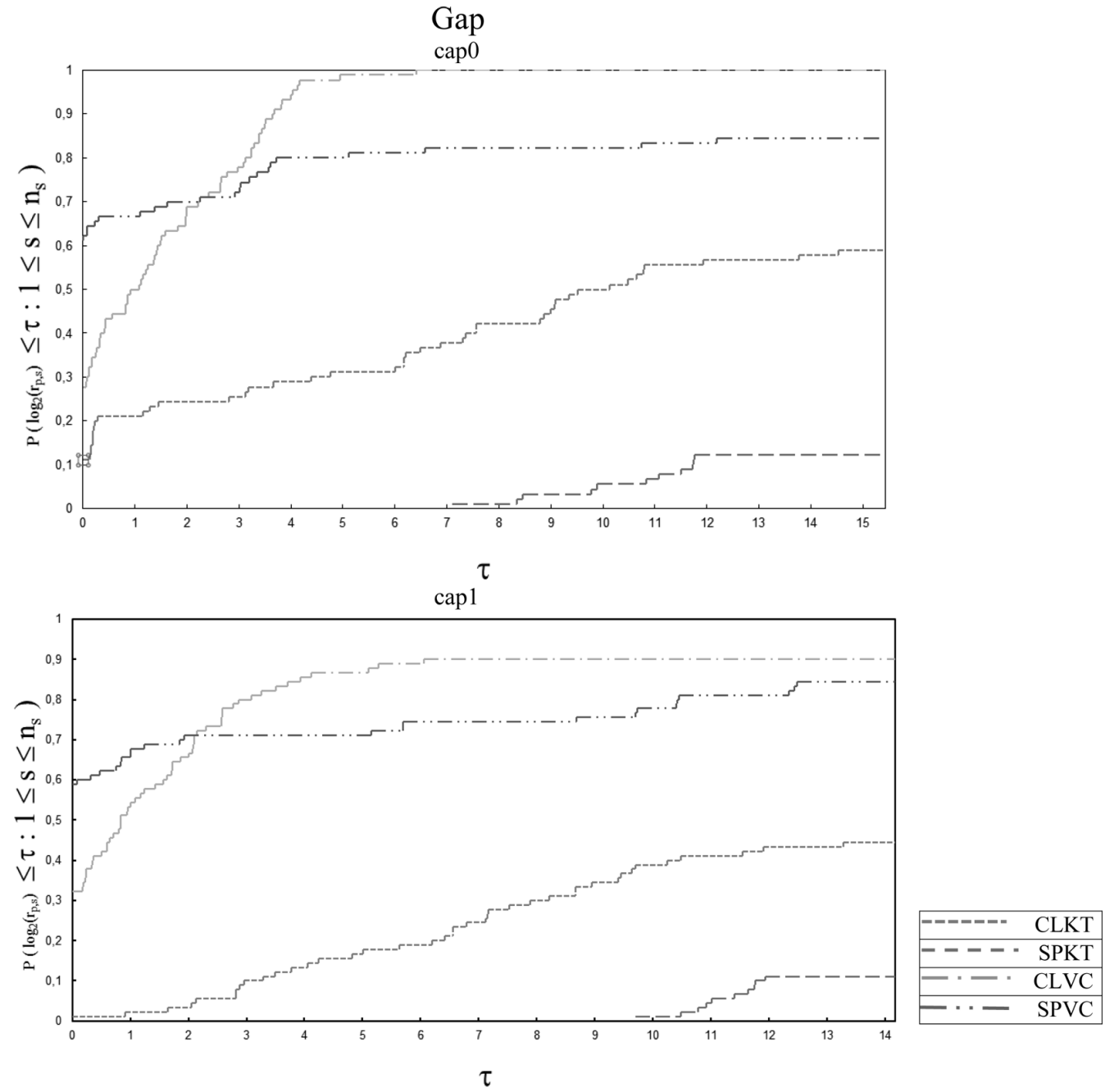

Figure 3 - Performance Profiles: Gap for Optimization Package Based Heuristic - Data 1.

\subsection{Experiment 2}

The following describes the generation of data and computational results for the integrated uncapacitated lot-sizing and the cutting stock problem with several types of objects (the models in Section 4 and its corresponding model in Section 5).

\subsubsection{Data Set}

The proposed data set is based on Poldi \& Arenales (2010) and for the lot-sizing problem the data is based on Trigeiro et al. (1989), but without considering capacity constraints. 
Table 5 - LP Relaxation for Data 2.

\begin{tabular}{|c|c|c|c|c|}
\hline Class 10 & $C L K T$ & $S P K T$ & $C L V C$ & $S P V C$ \\
\hline Example 1 & 12552.56 & 38450.41 & 12552.81 & 38455.10 \\
Example 2 & 12534.92 & 38645.79 & 12535.19 & 38649.20 \\
Example 3 & 25058.61 & 74650.39 & 25058.67 & 74651.33 \\
Example 4 & 46554.59 & 143706.15 & 46554.59 & 143706.16 \\
Example 5 & 13311.81 & 63092.75 & 13312.38 & 63096.81 \\
Example 6 & 30428.71 & 136060.92 & 30428.72 & 136062.38 \\
Example 7 & 64483.84 & 299427.41 & 64483.84 & 299427.59 \\
\hline Average & 29275.01 & 113433.40 & 29275.17 & 113435.51 \\
\hline \hline Class 11 & \multicolumn{5}{|c}{} \\
\hline Example 1 & 14831.41 & 40729.26 & 15257.52 & 41292.36 \\
Example 2 & 14813.77 & 40924.64 & 15243.59 & 41476.43 \\
Example 3 & 29619.41 & 79211.19 & 30346.19 & 80104.87 \\
Example 4 & 56385.07 & 153536.63 & 57257.32 & 154658.84 \\
Example 5 & 18060.64 & 67841.58 & 18919.93 & 68835.03 \\
Example 6 & 40162.36 & 145794.58 & 41623.78 & 147495.46 \\
Example 7 & - & - & 86738.85 & 322311.95 \\
\hline Average & - & - & 37912.46 & 122310.71 \\
\hline \hline Class 12 & \multicolumn{5}{|c|}{-16508.34} & 42542.73 \\
\hline Example 1 & 15702.19 & 41600.04 & 16493.35 & 42720.26 \\
Example 2 & 15684.55 & 41795.42 & 16498.93 & 125845.83 \\
Example 3 & 31270.20 & 80861.98 & 32472.08 & 82150.89 \\
Example 4 & 59625.74 & 156777.30 & 61617.88 & 158934.87 \\
Example 5 & 19827.17 & 69608.12 & 21501.31 & 71404.10 \\
Example 6 & 43685.89 & 149318.11 & 46154.93 & 152056.45 \\
Example 7 & - & - & 95674.65 & 331111.52 \\
\hline Average & - & - & 41488.9 \\
\hline
\end{tabular}

(-) time limit with no lower bound

- number of periods: $T=\{3,6\}$

- types of objects: $K=\{3,5\}$

- number of items: $I=\{10,20\}$

- object length: $L_{k} \in[300,1000]$

- planned supply of object type $k$ in period $t: e_{k t} \in\left[1.5\left\lceil\lambda_{t}\right\rceil, 2\left\lceil\lambda_{t}\right\rceil\right]$ with, $\lambda_{t}=\frac{\sum_{i=1}^{I} l_{i} d_{i t}}{\sum_{k=1}^{K} L_{k}}$.

- item length: $l_{i} \in[0,1 \bar{L}, 0,4 \bar{L}]$, whith $\bar{L}=\frac{\sum_{k=1}^{K} L_{k}}{K}$

- item demand: $d_{i t} \in[10,200]$ 

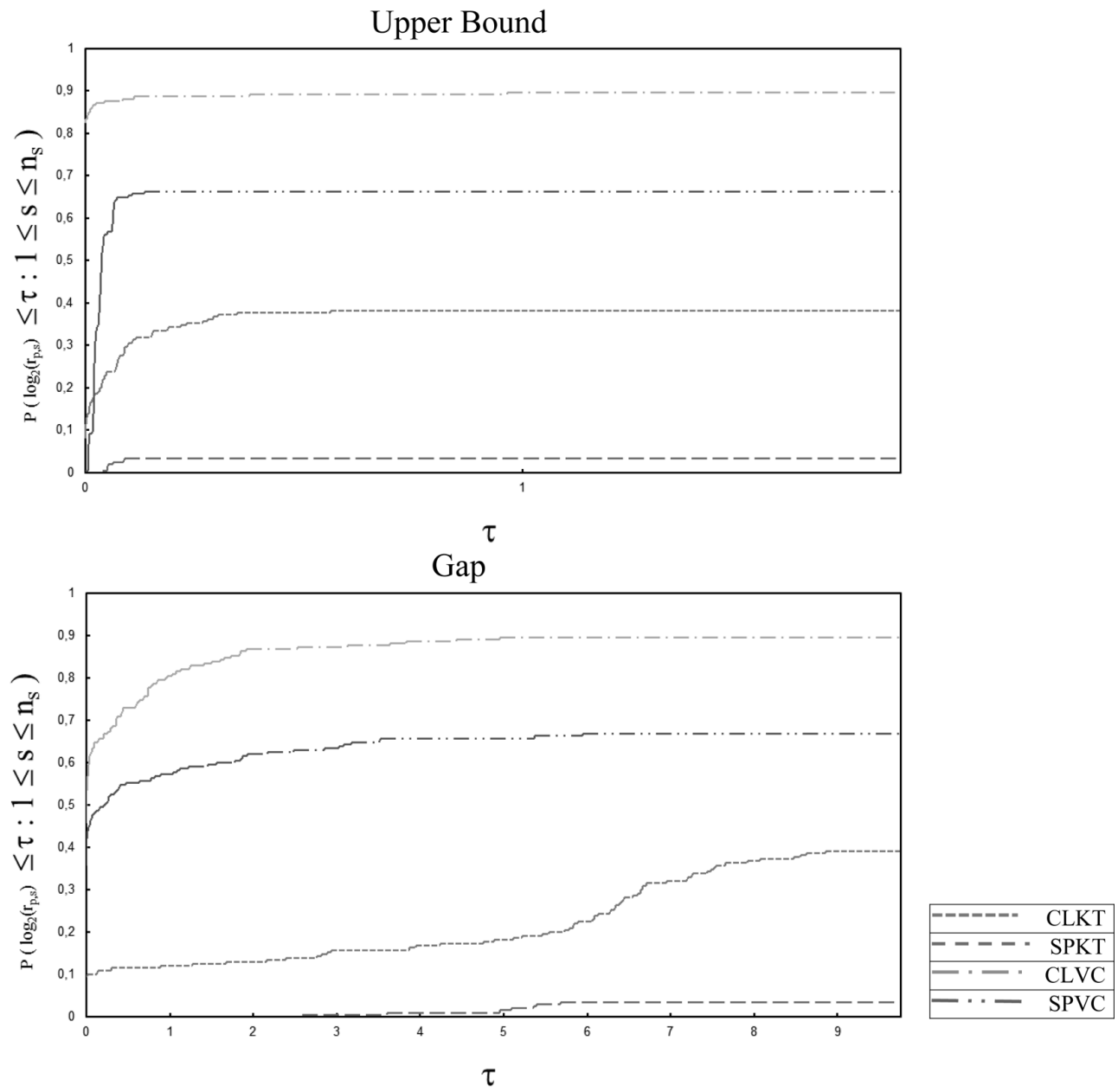

Figure 4 - Performance Profiles: Upper Bound for the Optimization Package Based Heuristic - Data 2.

- raw material cost: $c w=1$;

- setup cost: $s c_{i t} \in[100,500]$

- inventory cost: $h c_{i t} \in[1,5]$

Thus, the data set defines 8 classes (see Table 6), and for each class 10 random instances are generated, totalling 80 instances.

\subsubsection{Computational Results}

This data set included 3 infeasible instances (Class 16 instance 10 and Class 20 instances 5 and $10)$ and these were removed from the analysis. 


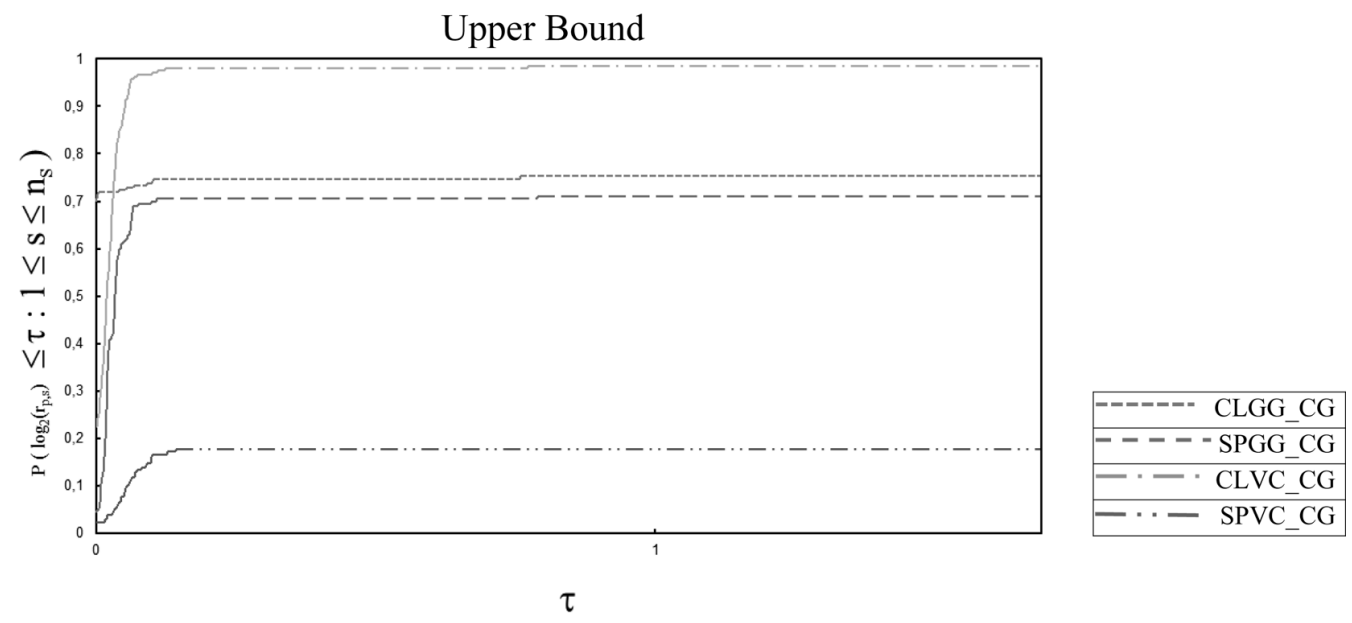

Figure 5 - Performance Profiles: Upper Bound for the Column Generation Based Heuristic - Data 2.

Table 6 - Classes with several types of objects.

\begin{tabular}{|c|c|c|c|}
\hline Classes & Periods & Objects & Items \\
\hline Class 13 & 3 & 3 & 10 \\
Class 14 & 3 & 3 & 20 \\
Class 15 & 3 & 5 & 10 \\
Class 16 & 3 & 5 & 20 \\
Class 17 & 6 & 3 & 10 \\
Class 18 & 6 & 3 & 20 \\
Class 19 & 6 & 5 & 10 \\
Class 20 & 6 & 5 & 20 \\
\hline
\end{tabular}

Table 7 presents the computational results obtained for the lower bound. The use of the reformulated lot-sizing model shows significant improvements in the lower bound. A slight improvements can be seen in the use of $V C$ model when compared with the KT model.

Tables 8 and 9 show the average upper bounds in each class for each mathematical model. TheWWKTMO and SPKTMO models did not find a feasible solution for most classes. Considering the optimization package based heuristic the WWVCMO model found the best results for almost all the classes (except Classe 16). For the column generation based heuristic the best results are shared between WWGGMO_CG and SPGGMO_CG models. As the WWGGMO_CG model obtained results which are very close to the best found, this fact contributed to the best global average. Note that $W W V C M O$ performs very poorly on Class 20, compared to the column generation methods. This is also confirmed by the largest gap for this class.

Table 10 presents the average for the gap obtained in each class for models solved with the optimization package based heuristic. For this strategy the gap used is that provided by the solver 
Table 7 - LP Relaxation.

\begin{tabular}{|c|c|c|c|c|}
\hline Classes & WWKTMO & WWVCMO & SPKTMO & SPVCMO \\
\hline Class 13 & 4861.68 & 4861.68 & 5472.80 & 5472.80 \\
Class 14 & 9840.43 & 9840.43 & 9931.05 & 9931.05 \\
Class 15 & 4801.52 & 4868.99 & 5201.06 & 5201.06 \\
Class 16 & 9872.04 & 9873.19 & 9872.04 & 9873.19 \\
Class 17 & 6831.39 & 6831.39 & 8743.99 & 8779.76 \\
Class 18 & 13455.68 & 13455.68 & 13510.54 & 13510.54 \\
Class 19 & 6584.89 & 6584.89 & 7436.32 & 7436.32 \\
Class 20 & 13626.16 & 13637.07 & 13626.16 & 13637.07 \\
\hline Average & 8734.22 & 9225.75 & 8742.66 & 9230.23 \\
\hline
\end{tabular}

Table 8 - Upper Bounds for the Optimization Package Based Heuristic.

\begin{tabular}{|c|c|c|c|c|}
\hline Classes & WWKTMO & WWVCMO & SPKTMO & SPVCMO \\
\hline Class 13 & 18014.40 & $\mathbf{8 0 8 1 . 2 0}$ & 40179.80 & 8081.70 \\
Class 14 & $(* 5)$ & $\mathbf{1 5 1 8 8 . 2 0}$ & - & 15214.40 \\
Class 15 & 17307.50 & $\mathbf{7 6 2 9 . 4 0}$ & 30391.60 & 7629.90 \\
Class 16 & $(* 6)$ & 14634.00 & - & $\mathbf{1 4 6 2 7 . 6 7}$ \\
Class 17 & $(* 4)$ & $\mathbf{1 6 4 4 7 . 1 0}$ & - & 16507.30 \\
Class 18 & $(* 2)$ & $\mathbf{2 8 8 0 1 . 3 0}$ & - & 29546.30 \\
Class 19 & $(* 5)$ & $\mathbf{1 5 5 6 0 . 8 0}$ & - & 15721.90 \\
Class 20 & - & $\mathbf{2 1 9 0 4 8 . 6 3}$ & - & $(* 1)$ \\
Average & - & $\mathbf{4 0 6 7 3 . 8 3}$ & - & - \\
\hline
\end{tabular}

(-) time limit with no integer solution

(*) number of instances with feasible solution

Table 9 - Upper Bounds for the Column Generation Based Heuristic.

\begin{tabular}{|c|c|c|c|c|}
\hline Classes & $W W G G M O_{-} C G$ & $W W V C M O_{-} C G$ & SPGGMO_CG & SPVCMO_CG \\
\hline Class 13 & 8171.40 & 8509.50 & $\mathbf{8 1 6 1 . 4 0}$ & 8456.80 \\
Class 14 & $\mathbf{1 5 4 4 0 . 9 0}$ & 20884.90 & 15442.40 & 21045.90 \\
Class 15 & 7586.40 & 9196.00 & $\mathbf{7 5 7 7 . 2 0}$ & 9373.10 \\
Class 16 & $\mathbf{1 4 9 4 1 . 0 0}$ & 22470.22 & 14999.89 & 22707.78 \\
Class 17 & $\mathbf{1 6 6 1 6 . 1 0}$ & 19200.20 & 16675.70 & 19736.20 \\
Class 18 & $\mathbf{2 9 9 9 6 . 2 0}$ & 47068.00 & 33889.80 & 51973.40 \\
Class 19 & 15335.90 & 19712.30 & $\mathbf{1 5 3 3 4 . 6 0}$ & 20369.80 \\
Class 20 & $\mathbf{3 2 3 4 0 . 5 0}$ & 45904.88 & 52861.25 & 47941.50 \\
Average & $\mathbf{1 7 5 5 3 . 5 5}$ & 24118.25 & 20617.78 & 25200.56 \\
\hline
\end{tabular}

for the final solution. As can be seen, most of the best gaps are found by WWVCMO model. In general, the influence of data on the results relates to the increase in the number of items (in the even-numbered classes), where the gaps are significantly higher compared to the odd-numbered classes. 
Table 10 - Final Gap.

\begin{tabular}{|c|c|c|c|c|}
\hline Classes & WWKTMO & WWVCMO & SPKTMO & SPVCMO \\
\hline Classe 13 & 58.79 & $\mathbf{0 . 1 0}$ & 80.41 & 0.11 \\
Classe 14 & $(* 5)$ & $\mathbf{0 . 2 9}$ & - & 0.46 \\
Classe 15 & 58.94 & $\mathbf{0 . 0 8}$ & 74.78 & 0.08 \\
Classe 16 & $(* 6)$ & 0.39 & - & $\mathbf{0 . 3 5}$ \\
Classe 17 & $(* 4)$ & 0.24 & - & $\mathbf{0 . 2 2}$ \\
Classe 18 & $(* 2)$ & $\mathbf{2 . 7 8}$ & - & 3.96 \\
Classe 19 & $(* 5)$ & $\mathbf{0 . 5 0}$ & - & 1.55 \\
Classe 20 & - & $\mathbf{4 8 . 1 6}$ & - & $(* 1)$ \\
Average & - & $\mathbf{6 . 5 7}$ & - & - \\
\hline
\end{tabular}

\section{CONCLUSIONS}

In this paper, we present a study of mathematical models from the literature for modeling the lotsizing problem and the one-dimensional cutting stock problem, in order to propose and compare new integrated problems. For the lot-sizing problem, we study the model proposed by Wagner \& Whitin (1958), the model proposed by Trigeiro et al. (1989) and a reformulation of the lotsizing problem proposed by Eppen \& Martin (1987). For the cutting stock problem, extensions of the models proposed by Kantorovich (1960), Valério de Carvalho $(1999,2002)$ with reduction criteria and Gilmore \& Gomory (1961) have been proposed to incorporate multiple periods. These models have been extended also to consider various types of objects.

In the literature, there are several studies of practical cases, which use these formulations to model the problems found, as simple or integrated problems. This study aims to present alternative mathematical models for the integrated problem in order to compare and point out the advantages of each model, as well as, the impact of the data set on the obtained solutions. As solution methods, we present two strategies. The first one uses a solver for finding the solution. In the second one, the column generation technique is used in a heuristic strategy to get a feasible solution. An extensive computational study is conducted with different data sets.

The difficulty of the models to obtain a feasible solution becomes clear when considering the capacity constraint in the lot-sizing problem, and this impact is even greater when the capacity constraint is tight. The main influence of the data set on the results is in instances that have an item length considerably smaller when compared to the object length. For the models that consider various object types in stock, the main impact of the data in the results comes from the increase in the number of items.

In general, models integrated with the classical lot-sizing problem and the cutting stock problem model based on Valério de Carvalho $(1999,2002)$ with column generation obtained the largest number of feasible solutions compared to other mathematical models in all analyses. It is also possible to generalize the fact that the models that integrate with the model proposed by Kantorovich (1960) have produced bad results in all experiments. For models with capacity 
constraints, the classical lot-sizing model integrated with the Valério de Carvalho model and Gilmore and Gomory in both capacity variations (uncapacitated model and $\operatorname{cap}_{t}=\operatorname{cap}_{t} / 0,3$ ) obtained a lot of good upper bounds. However the use of the shortest path model (Eppen \& Martin, 1987) significantly improved the $L P$ lower bound and obtained gaps smaller when compared to other models, for most classes. In an analysis for the mathematical models with various object types, although the integration with the Gilmore and Gomory model does not show the best results for all classes for the upper bound, the proximity to the best results contributes to the achievement of best overall average.

A possible direction for future research is to study other extensions of the models proposed and develop more elaborate solution methods, in order to address a greater variety of instances.

\section{ACKNOWLEDGEMENTS}

We would like to thank the anonymous referees for their constructive comments, which led to a clearer presentation of the material. This research was funded by the Conselho Nacional de Desenvolvimento Científico e Tecnológico - CNPq and the Fundação de Amparo a Pesquisa do Estado de São Paulo - FAPESP (process no. 474782/2013-1, 2012/20631-2, 2014/17273-2 and 2014/01203-5).

\section{REFERENCES}

[1] Alem D \& Morabito R. 2013. Risk-averse two-stage stochastic programs in furniture plants. $O R$ Spectrum, 35(4): 773-806, 2013. ISSN 0171-6468.

[2] Alem DJ \& Morabito R. 2012. Production planning in furniture settings via robust optimization. Computers \& Operations Research, 39(2): 139-150.

[3] De Araujo SA, de Reyck B, Degraeve Z, Fragkos I \& Jans R. 2015. Period decompositions for the capacitated lot sizing problem with setup times. INFORMS Journal on Computing, 27(3): 431-448.

[4] Degraeve $Z$ \& Jans R. 2007. A new dantzig-wolfe reformulation and branch-and-price algorithm for the capacitated lot-sizing problem with setup times. Operations Research, 55(5): 909-920.

[5] Degraeve Z \& Peeters M. 2003. Optimal integer solutions to industrial cutting-stock problems: Part 2, benchmark results. INFORMS Journal on Computing, 15(1): 58-81.

[6] Denizel M, Altekin FT, SÜral H \& Stadtler H. 2008. Equivalence of the lp relaxations of two strong formulations for the capacitated lot-sizing problem with setup times. OR Spectrum, 30(4): 773-785.

[7] Dolan ED \& MoRÉ JJ. 2002. Benchmarking optimization software with performance profiles. Mathematical Programming, 91(2): 201-213.

[8] EPPEN GD \& MARTIN RK. 1987. Solving multi-item capacitated lot-sizing problems using variable redefinition. Operations Research, 35(6): 832-848.

[9] FARLEY AA. 1988. Mathematical programming models for cutting-stock problems in the clothing industry. The Journal of the Operational Research Society, 39(1): 41-53. 
[10] Fiorotto DJ \& De Araujo SA. 2014. Reformulation and a lagrangian heuristic for lot sizing problem on parallel machines. Annals of Operations Research, 217(1): 213-231.

[11] Fourer R, GAy DM \& Kernighan BW. 1990. A modeling language for mathematical programming. Management Science, 36(5): 519-554.

[12] GaU T \& W ÄSChER G. 1995. Cutgen1: A problem generator for the standard one-dimensional cutting stock problem. European Journal of Operational Research, 84(3): 572-579.

[13] Ghidini CTLS \& ARenales MN. 2009. Otimização de processos acoplados na indústria de móveis: dimensionamento de lotes e corte de estoque. In Anais do CNMAC-Congresso Nacional de Matemática Aplicada e Computacional.

[14] Ghidini CTlS, Alem D \& Arenales MN. 2007. Solving a combined cutting stock and lotsizing problem in small furniture industries. In Proceedings of the 6th International Conference on Operational Research for Development (VI-ICORD).

[15] Gilmore PC \& GOMORY RE. 1961. A linear programming approach to the cutting-stock problem. Operations Research, 9(6): 849-859.

[16] Gilmore PC \& Gomory RE. 1963. A linear programming approach to the cutting stock problema - part ii. Operations Research, 11(6): 863-888.

[17] GRamani MCN \& FranÇA PM. 2006. The combined cutting stock and lot-sizing problem in industrial processes. European Journal of Operational Research, 174(1): 509-521.

[18] Gramani MCN, França PM \& Arenales MN. 2009. A lagrangian relaxation approach to a coupled lot-sizing and cutting stock problem. International Journal of Production Economics, 119(2): 219-227.

[19] Gramani MCN, França PM \& Arenales MN. 2011. A linear optimization approach to the combined production planning model. Journal of the Franklin Institute, 348(7): 1523-1536.

[20] Hendry LC, FoK KK \& SHeK KW. 1996. A cutting stock and scheduling problem in the copper industry. The Journal of the Operational Research Society, 47(1): 38-47.

[21] IBM CORPORATION. 2009. CPLEX. http://www-01.ibm.com/software/integration/optimization/ cplex-optimizer/, 2009. visitada pela última vez em 12/10/2012.

[22] JANS R. 2009. Solving lot-sizing problems on parallel identical machines using symmetry-breaking constraints. INFORMS Journal on Computing, 21(1): 123-136.

[23] JANS R \& DEgRAEVE Z. 2004. Improved lower bounds for the capacitated lot sizing problem with setup times. Operations Research Letters, 32(2): 185-195.

[24] Kantorovich LV. 1960. Mathematical methods of organizing and planning production. Management Science, 6(4): 366-422.

[25] LeÃo APS \& Arenales MN. 2012. Análise de modelos matemáticos para o problema de corte de estoque unidimensional acoplado ao dimensionamento de lotes. In Anais do XLIV SBPO-Simpósio Brasileiro de Pesquisa Operacional, Rio de Janeiro-RJ.

[26] Longhi AL, Melega GM \& de Araujo SA. 2015. Modelos matemáticos para o problema integrado de dimensionamento de lotes e corte de estoque unidimensional. Pesquisa Operacional para o Desenvolvimento, 7(1): 82-104. 
[27] Melega GM, Fiorotto DJ \& De Araujo SA. 2013. Formulações fortes para o problema de dimensionamento de lotes com várias plantas. TEMA (São Carlos), 14: 305-318. ISSN 2179-8451.

[28] Molina F, Morabito R \& de Araujo SA. 2016. Mip models for production lot-sizing problems with distribution costs and cargo arrangement. Journal of the Operational Research Society. Accept to Publication.

[29] NonÅs SL \& THORSTENSON A. 2000. A combined cutting-stock and lot-sizing problem. European Journal of Operational Research, 120(2): 327-342.

[30] NonÅs SL \& Thorstenson A. 2008. Solving a combined cutting-stock and lot-sizing problem with a column generating procedure. Computers \& Operations Research, 35(10): 3371-3392.

[31] Pochet Y \& Wolsey LA. 2006. Production planning by mixed integer programming. Springer Science \& Business Media.

[32] Poldi KC \& Arenales MN. 2010. O problema de corte de estoque unidimensional multiperíodo. Pesquisa Operacional, 30: 153-174.

[33] Poldi KC \& de Araujo SA. 2016. Mathematical models and a heuristic method for the multiperiod one-dimensional cutting stock problem. Annals of Operations Research, 238(1): 497-520.

[34] Poltroniere SC, Poldi KC, Toledo FMB \& Arenales MN. 2008. A coupling cutting stocklot sizing problem in the paper industry. Annals of Operations Research, 157(1): 91-104.

[35] SAntos SG, DE ARAujo SA \& RANGEL MSN. 2011. Integrated cutting machine programming and lot sizing in furniture industry. Pesquisa Operacional para o desenvolvimento, 3(1): 1-17.

[36] Silva E, Alvelos F \& Valério de Carvalho JM. 2014. Integrating two-dimensional cutting stock and lot-sizing problems. Journal of the Operational Research Society, 65(1): 108-123.

[37] Trigeiro WW, Thomas LJ \& MCClain JO. 1989. Capacitated lot sizing with setup times. Management Science, 35(3): 353-366.

[38] VAlÉrio de Carvalho JM. 1999. Exact solution of bin-packing problems using column generation and branch-and-bound. Annals of Operations Research, 86: 629-659.

[39] VALÉRIO DE CARVALHO JM. 2002. Lp models for bin packing and cutting stock problems. European Journal of Operational Research, 141(2): 253-273.

[40] Vanzela M, Rangel S \& DE Araujo SA. 2013. The integrated lot sizing and cutting stock problem in a furniture factory. In Intelligent Manufacturing Systems, volume 11, pages 390-395.

[41] Vyve MV \& Wolsey LA. 2006. Approximate extended formulations. Mathematical Programming, 105(2-3): 501-522.

[42] Wagner HM \& Whitin TM. 1958. Dynamic version of the economic lot size model. Management Science, 5(1): 89-96. 